\title{
BMJ Open Magnetoencephalography to investigate central perception of exercise-induced breathlessness in people with chronic lung disease: a feasibility pilot
}

\author{
Miriam J Johnson, ${ }^{1}$ Michael IG Simpson, ${ }^{2}$ David C Currow, ${ }^{3}$ Rebecca E Millman, ${ }^{2}$ \\ Simon P Hart, ${ }^{1}$ Gary Green ${ }^{1,2,4}$
}

To cite: Johnson MJ, Simpson MIG, Currow DC, et al.

Magnetoencephalography to investigate central perception of exercise-induced breathlessness in people with chronic lung disease: a feasibility pilot. BMJ Open 2015;5: 007535 .

doi:10.1136/bmjopen-2014007535

- Prepublication history and additional material is available. To view please visit the journal (http://dx.doi.org/ 10.1136/bmjopen-2014007535).

Received 23 December 2014 Revised 17 April 2015 Accepted 8 May 2015

\section{(a) CrossMark}

${ }^{1}$ Hull York Medical School, University of Hull, Hull, UK ${ }^{2}$ York Neuroimaging Centre, University of York, York, UK ${ }^{3}$ Discipline, Palliative and Supportive Services, Flinders University, Adelaide, Australia ${ }^{4}$ Department of Psychology, University of York, York, UK

Correspondence to

Professor Miriam J Johnson; miriam.johnson@hyms.ac.uk

\section{ABSTRACT}

Objectives: Neuroimaging in chronic breathlessness is challenging. The study objective was to test the feasibility of magnetoencephalography (MEG) for functional neuroimaging of people with chronic breathlessness.

Design: Feasibility pilot study.

Setting: Respiratory clinic out-patients.

Participants: 8 patients (mean age $=62$; (range 47-83); 4 men) with chronic non-malignant lung disease; modified MRC breathlessness score $\geq 3$ (median $\mathrm{mMRC}=4$ ), intensity of exercise-induced breathlessness $>3 / 10$; no contraindication to MRI scanning.

Methods and measures: 4 MEG scans were conducted for each participant: (1) at rest (5 mins), (2) postseated leg exercise-induced breathlessness during recovery (10 mins). Recovery scans (2) were conducted with/without facial airflow in random order; both scans were repeated $1 \mathrm{~h}$ later. Participants rated breathlessness intensity (0-10 Numerical Rating Scale (NRS)) at baseline, maximal exertion and every minute during recovery, and rated acceptability of study procedures at the end of the study (0-10 NRS). A structural MRI scan was conducted for MEG coregistration and source-space analyses. Rest data were compared with data from healthy volunteers $(\mathrm{N}=6$; 5 men; mean age $=30.7$ years \pm 3.9 years).

Results: Exercises and MEG scanning were acceptable to all participants; 7/8 completed the MRI scans.

Maximum breathlessness intensity was induced by 5 min' exercise. The same level was induced for repeat scans (median=8; IQR=7-8). All recovered to baseline by $10 \mathrm{~min}$. Time-frequency profiles of data from the first and last 3 min were analysed in MEG source space based on breathlessness location estimates. Source localisation was performed, but anatomical source inference was limited to the level of the lobe. Differences in areas of activity were seen: during recovery scans; with and without airflow; and between participants/ normal volunteers at rest.

Conclusions: MEG is a feasible method to investigate exercise-induced breathlessness in people breathless with chronic lung disease, and able to identify neural activity related to changes in breathlessness.

\section{Strengths and limitations of this study}

- Participants with breathlessness due to chronic lung disease tolerated the isometric exercise and scanning procedures for this study.

- MEG is able to identify neural activity related to changes in breathlessness which can be induced by physical exertion.

- MEG appears to be a feasible method to investigate this challenging clinical problem.

- These data are preliminary and caution should be applied regarding the interpretation of the neuroimaging findings.

- Further work is needed with age-matched controls and using a larger cohort to confirm these findings.

\section{INTRODUCTION}

Despite treatment of the underlying condition(s), refractory breathlessness is a common, devastating consequence of diseases such as chronic obstructive pulmonary disease (COPD), cancer and heart failure. ${ }^{12}$

Headway has been made in the delineation of peripheral pathways and sensory afferent sources of respiratory sensation. ${ }^{3}$ However, there is growing interest in central nervous system pathways as the increased drive to breathe in people with breathlessness involves both brainstem and cortical processes. ${ }^{4}$ Positron emission tomography (PET) scanning and functional MRI (fMRI) have helped elucidate the integrated cortical and subcortical network involved in perception of, and response to, breathlessness by inducing unpleasant respiratory sensations in health volunteers. ${ }^{5} 6$

Little is known of how exercise-induced breathlessness is centrally processed in people with chronic lung diseases. Studies suggest that asthma patients demonstrate habituation and downregulation of perceived 
unpleasantness due to breathlessness; ${ }^{7}$ people with a longer duration of asthma have larger periaqueductal grey areas, ${ }^{8}$ and there are differences in the pattern of brain stem activity in the caudal ventro-lateral pons (parafacial group), an area relating to respiratory rhythmogenesis, between healthy volunteers and people with COPD. ${ }^{9}$

Work to date on mechanisms of breathlessness has largely been undertaken in healthy volunteers. Methods of inducing breathlessness in healthy volunteers seem adequate to model aspects of acute breathlessness which resolve quickly, but, as the signal becomes unstable after a few seconds, investigation of chronic breathlessness may be more limited. ${ }^{4}$

There is little published work to explore the central mechanisms of interventions on breathlessness in people with lung disease. ${ }^{4}$ The choice of functional imaging in people with chronic lung disease for whom breathlessness is induced with exercise is limited by technical factors such as (1) the ability to lie flat in an MRI scanner for the duration required for a functional study and (2) the ability to perform exertion to induce breathlessness while lying flat in the scanner. It is possible that techniques such as MRI-compatible ergometry may be a potential method of investigation allowing exertioninduced breathlessness, but the patient is still required to lie flat.

\section{Magnetoencephalography}

Magnetoencephalography (MEG) is, possibly, a better tolerated but untried method of functional brain imaging in people with limited exercise tolerance and restricted positioning due to lung disease. Participants are seated within the scanner and neuronal activity measured directly rather than changes in blood flow in response to the activity. ${ }^{4}$ MEG scanning has been highlighted as a rapidly developing and unique tool for the investigation of brain activity. ${ }^{10}$ MEG may be useful in preference to EEG because of the superior sourcelocalisation abilities of MEG.

More detailed description can be found elsewhere, ${ }^{10} 11$ but in summary, neuronal activity generates small magnetic fields perpendicular to the dendritic current flow. The small magnetic fields pass through the skull and are measured in the MEG scanner using superconducting quantum interference device magnetometers in a helmet-shaped sensor holder. Structural MRI scans are required to delineate the source of the brain activity measured in MEG, taking only a few minutes with a $3 \mathrm{~T}$ scanner.

\section{Cool airflow in refractory breathlessness}

Clinical and emerging research evidence indicates that cool facial airflow may reduce breathlessness. ${ }^{12-14}$ In people with mild or no hypoxaemia, the passage of air over the nasal mucosa using a hand-held, batteryoperated fan may provide the same symptomatic benefit as oxygen therapy. ${ }^{15}$ This intervention is universally available, cheap, non-invasive, has no contraindications, and is easy to use alongside other interventions. To date, there have been no studies of the central neuronal impact of a hand-held fan.

Study aims: The study aimed to test the feasibility of MEG scanning as a tool for the investigation of refractory breathlessness due to chronic respiratory disease with regard to:

- Participant acceptability of procedures;

- Whether activity in areas of the brain that are known to mediate breathlessness are identified by MEG/MRI at rest, following exertion, and upon recovery; and

- Whether such activity is further modulated by airflow across the face.

As this had not been previously attempted, a pilot was necessary to establish the technical, administrative and logistic feasibility of a full-scale study.

\section{METHODS}

Ethical approval was obtained from the National Research Ethical Service Committee Yorkshire and the Humber-Leeds East and the York Neuro-imaging Centre, University of York. NHS permission was obtained from the Hull and East Yorkshire Hospitals NHS Trust Research and Development Department.

\section{Participants and setting}

A pilot cohort was formed of consenting adults recruited from a single academic respiratory centre. Eligible patients were living independently in their own home, and had modified Medical Research Council (mMRC) breathlessness score 3 (stop for breath after walking for $100 \mathrm{~m}$, or after a few minutes, on the level), or 4 (too breathless to leave the house, or breathless when dressing) due to a chronic respiratory disease. Participants were estimated to develop at least moderate breathlessness ( $>3$ on a $0-10$ numerical rating scale) by 5 min of seated exercise. Exclusions included participants who had taken opioid medication within the previous $24 \mathrm{~h}$, or who were ineligible for MRI scanning, including significant claustrophobia or orthopnoea.

\section{Data collection}

Baseline data included primary cause and duration of breathlessness, age and sex, and mMRC breathlessness score. As the purpose of this study was to establish feasibility of procedures and data analysis, baseline data did not include a more detailed phenotyping of respiratory compromise in order to minimise participant burden, and because physiological measures such as forced expiratory volume in 1 second $\left(\mathrm{FEV}_{1}\right)$ do not correlate well with perceived breathlessness.

Exercise and scans

Four MEG scans were conducted (figure 1). 
Step 1

Participant prepared in MEG scanner

Step 2

NRS score: breathlessness intensity at rest

MEG scan (\#1 and \#3): 5 minutes rest

Step 3

Exercise: maximum 5 minutes

NRS score: breathlessness intensity maximum tolerated exertion

Step 4

Rest from exertion
MEG scan (\#2 and \#4): 10 minutes recovery
At least an hour's break
every minute
Repeat steps 1 to 4
Structural MRI scan

Figure 1 Flow chart: scanning schema.

- Following an initial 5 min MEG scan at rest, participants then performed a seated leg exercise using a physiotherapy band until maximal tolerated breathlessness or $5 \mathrm{~min}$ of exercise, whichever came first (box 1).

- As soon as the participant stopped exercising, MEG scanning recommenced during a recovery phase until NRS scores were stable for $3 \mathrm{~min}$ or a maximum of $10 \mathrm{~min}$ had elapsed. Ten minutes were allowed for recovery. ${ }^{16}$

\section{Box 1 Exercise protocol}

The patient was seated comfortably within the scanner and prepared for scanning.

- The patient verbally scored their breathlessness at rest (0-10 NRS).

- One end of an exercise resistance band was placed over the patient's non-dominant foot and the other end held by their dominant hand.

- Fixing one end of the band with their hand, the patient then exercised using their leg against resistance until they reached maximum tolerated breathlessness, or until a maximum of $5 \mathrm{~min}$, or the clinical researcher (MJ) advised them to stop.

- At maximum tolerated breathlessness, the patient gave a 0-10 NRS intensity rating and rested. MJ immediately left the MEG room and scanning commenced for the 10 min recovery phase.

- Heart rate was monitored continuously throughout the procedure (exercise and recovery).

- Pulse oximetry using a finger pulse oximeter was monitored during exercise only.
- Steps 1 to 4 were repeated after an hour during which the participant was given a break, out of the scanning room.

Recovery scans (scan \#2 and \#4) were conducted with or without facial cool airflow in random order using a tube directing air from a cylinder across the lower half of the face. Airflow was directed for the first 5 min of recovery.

A structural MRI scan was conducted after the MEG scans to enable anatomical inference during the source analysis.

Participants rated breathlessness intensity 'now' (0-10 numerical rating scale (NRS); $0=$ no breathlessness and $10=$ worst imaginable breathlessness) at baseline rest, maximal exertion, and then every minute during recovery. Participants rated the acceptability of the study procedures (NRS $0=$ totally unacceptable; $10=$ completely acceptable).

\section{Control group for the rest scans}

Data at rest were compared with previously acquired data from healthy volunteers held at the York Neuroimaging centre. Age or sex-matched controls were not available.

\section{MEG data acquisition}

MEG data were acquired using a 248 magnetometer 4D Neuroimaging Magnes 3600 system at the York Neuroimaging Centre at a sample rate of $678.17 \mathrm{~Hz}$, and online low-pass filtered at $200 \mathrm{~Hz}$. Three bad sensors were identified and excluded from all levels of data analysis. Head locations before and after the scan were recorded using five fiducial markers on the participant's face. Visible movement of the participant's heads with respiration was observed while the locations of the five fiducial landmarks were being recorded at the start of the scan, and throughout the duration of at least the first $5 \mathrm{~min}$. Head movement was calculated by the 4-D Neuroimaging software as the difference between the head coil positions at the start and end of each scan. Average head movement at the end of the rest scan was $0.32 \mathrm{~cm}( \pm 0.35 \mathrm{~cm})$, and after exercise was $0.7 \mathrm{~cm} \quad( \pm 0.51 \mathrm{~cm})$. The 4-D Neuroimaging system did not allow continuous localisation of head position relative to the MEG Dewar.

High-resolution T1 anatomical MRIs were acquired on a GE 3 T Excite HDx scanner at the York Neuroimaging Centre. The MRI were acquired with a reconstructed $1.5 \mathrm{~mm}$ isotropic resolution to minimise the duration. The location of the five fiducial landmarks, along with a headshape, were digitised using a Polhemus Fastrack 3D digitisation unit and coregistered with the T1 structural scan using a surface matching algorithm. ${ }^{17}$ Individual anatomical $\mathrm{T} 1$ structural MR scans were spatially normalised to the standard Montreal Neurological Institute (MNI) 152 brain, ${ }^{18}$ using the Oxford Centre for Functional Magnetic Resonance Imaging of the Brain (fMRIB) linear image registration tool (FLIRT) transform in FMRIB Software Library (FSL). ${ }^{19}$ These 
transform values were used to seed and report analyses in MNI coordinates.

\section{Sample size}

Given the exploratory nature of this cohort and the degree of breathlessness of the participants, data were reviewed in an ongoing manner to detect the point at which no further useful information was likely with regard to study objectives and, therefore, unethical to continue. This point was reached after seven completed scans.

\section{Analysis plan}

The data analysis was divided into two parts: resting state analysis; exercise analysis.

Visual inspection of $1 \mathrm{~s}$ epochs of the raw data was carried out to remove physiological (such as swallowing and blinking) and non-physiological artifacts before any data analysis took place.

\section{Resting state}

There were no movement-related issues, allowing anatomical statements. MEG data were source modelled using a vectorised linearly constrained minimum variance beamformer, ${ }^{20}$ using a multiple-spheres head model. ${ }^{21}$ A $5 \mathrm{~mm}$ grid was used for the beamforming analyses. Beamforming analyses create spatial filters to estimate the neural activity at each grid point in the brain. The outputs of beamformer spatial filters have been termed 'virtual electrodes'. ${ }^{22}$ In participants and in the normal volunteer data, virtual electrodes (VEs) were generated for locations in the dyspnoea pathway to generate a bandpass-filtered $(1-80 \mathrm{~Hz})$ power spectrum of the brain at rest. ${ }^{23}$

The seed MNI locations are: superior frontal gyrus $(-14,32,46)$; amygdala $(-24,-2,-22)$ anterior cingulate cortex $(-82,82,2)$; anterior insula $(-32,22,2)$; post $(-14,-36,64)$ and precentral gyrus $(-14,-30,60) .{ }^{23}$

To compare the activity in participants with lung disease with healthy volunteers, we calculated the non-phase-locked spectrum. Data in the 5 min resting acquisition were binned into $5 \mathrm{~s}$ epochs, and the spectrum of each epoch was calculated. These epoch-wise spectra were then averaged across epochs, and the subsequent average non-phase-locked spectra were then averaged across participants, and plotted with the respective $\mathrm{SE}$ of the mean.

\section{Exercise}

Using whole-head t-stat images, beamformer analysis was performed, but inference about the anatomical source was limited to the level of the lobe in which peaks of activity were observed. The first 3 min were assumed to represent activity during perceived breathlessness (supported by patient-report scores), and the last $3 \mathrm{~min}$ (all participants recovered to baseline level scores or stable to within 1 point) assumed to represent recovery: the first and last $3 \mathrm{~min}$ of recovery postexercise data were contrasted using a power contrast constrained within $\alpha$ $(8-13 \mathrm{~Hz}), \beta(13-30 \mathrm{~Hz})$ and $\gamma(30-80 \mathrm{~Hz})$ frequency bands, respectively. To ensure that no speech artifacts were included in the analysis, and that there were sufficient trials for statistical inference, the last $55 \mathrm{~s}$ of each minute were binned into $115 \mathrm{~s}$ epochs, resulting in $335 \mathrm{~s}$ epochs from the first $3 \mathrm{~min}$, being contrasted with $335 \mathrm{~s}$ epochs from the last $3 \mathrm{~min}$ of the postexercise scan. For each participant, in each frequency band, we performed a two-sample unpaired $t$ test between the power estimates from the first and last $3 \mathrm{~min}$ of data. To assess for significance, we performed a non-parametric shuffle permutation, where we permuted the labels of the epochs from the first and last $3 \mathrm{~min}$ of data 10000 times to generate a distribution of null t-statistics against which we compared the experimental data. To correct for multiple comparisons, only the maximum t-value across the source space was carried through for each permutation, to generate our null distribution. ${ }^{24}$ The top 5 peaks significant at $\mathrm{p}<0.05$, in each beamforming analysis, are reported in transformed MNI coordinates (see online supplementary table).

In view of the exploratory nature of the study, we also identified the MNI coordinates for brain activity that was statistically greater when breathless than after recovery, and vice versa.

\section{RESULTS}

Eight participants (mean age $=62$ (range 47-83); 4 men) completed all MEG scans, although one participant did not tolerate the MRI. Four participants had COPD, one of whom also had pulmonary sarcoid; three had asthma and one had bronchiectasis. One participant had an mMRC score of three and had been breathless between 1 and 5 years; all others scored 4 and had been breathless for over 5 years. At rest, two had no breathlessness and six were mildly breathless (NRS scores $<2$ ).

The control group consisted of data from six control subjects, and were included in the analyses, five of whom were men. The mean age was 30.7 years ( $\mathrm{std}= \pm 3.9$ years).

\section{Acceptability}

The MEG scans and leg exercises were acceptable to all participants (MEG: median NRS 10 (range 8-10); exercises: median 10 (range 7-10); completed MRI, median 10 (range 9-10); one not completing MRI scored 0). Maximum breathlessness intensity was induced by $5 \mathrm{~min}$, and induced to the same level for the repeat set of scans (median NRS intensity 8; IQR 7-8). All recovered to baseline, \pm 1 point by $10 \mathrm{~min}$. All had recovered to plateaued scores over 3 min within that timescale except for one participant who reported scores of 5, 3 and 1 during the last 3 min of data collection.

\section{Patient-reported breathlessness intensity with/without airflow}

Median time (minutes) to recovery was 5.5 for 'no airflow' (range 3-10; IQR 3.5-6.5) and 4.5 for 'airflow' 
(range 1-6; IQR 3-6). One participant had no difference in recovery time, but a technical problem caused imperceptible airflow. Several commented that the airflow was pleasant.

\section{MEG findings}

Predominant activity was in the $\beta(13-30 \mathrm{~Hz})$ and $\gamma(30-$ $80 \mathrm{~Hz}$ ) frequency bands. Preliminary beamformer-source localisation in individuals showed differences in areas of activity: between (1) participants/normal volunteers at rest; (2) postexercise/on recovery with/without airflow.

\section{Differences in group activity between participants and data from healthy volunteers at rest}

$\beta$ Activity at rest was statistically significantly greater in participants compared with normal controls. Figure 2 shows a consistent pattern in all the VEs in the second rest scan (to minimise potential artefact from unfamiliarity of the scanner). Initially, data from seven normal controls were used, but one with a similar increased $\beta$ wave activity was excluded on discovery that a respiratory condition had been diagnosed shortly after the scan had been performed.

\section{Differences within participant activity postexercise/ on recovery with/without airflow}

Brain activity which was significantly greater during postexertion breathlessness than on recovery, or conversely, greater on recovery than during postexertion breathlessness is presented in the online supplementary table. These results are summarised according to brain region of activity categorised as 'more than' or 'less than', by wave type $(\alpha, \beta$ or $\gamma)$, by breathlessness or recovery and by facial airflow or no facial airflow, in order to demonstrate patterns of activity (table 1). Figures 3 and 4 shows the $\alpha, \beta$ and $\gamma$ wave activity in participant $\# 05$ as a particularly clear example of the patterns seen in breathlessness/ recovery. Table 1 shows a group pattern of greater $\alpha$ and $\beta$ activity in the frontal and parietotemporal lobes during breathlessness compared to on recovery, particularly for frontal lobe activity. In addition, facial airflow appears to modify the parietotemporal $\alpha$ wave activity during breathlessness. $\alpha$ and $\beta$ activity share a pattern of reduced activity during breathlessness in the posterior regions, with greater activity seen after recovery. $\gamma$ Wave activity appears greater in breathlessness with airflow than without, and less in recovery with airflow than without.

\section{DISCUSSION}

MEG scanning followed by structural MRI was tolerated in a population with breathlessness on minimal exertion. All participants also found exercise to maximal tolerated breathlessness acceptable in the context of research.

Statistically significant differences in brain activity were found within individuals indicating that MEG scanning could be used to investigate central perception of breathlessness. When presented as categories of greater or less activity in the breathless or alternate recovered states, some group patterns in activity were seen. Different patterns of brain activity in breathlessness and relief is consistent with PET appearances in healthy volunteers; relief of breathlessness is associated with activity in different areas, not just an absence of activity in the areas associated with breathlessness perception. ${ }^{25}$ Furthermore, our data suggest that facial airflow may modify activity and reported perception.

Our feasibility study was not designed to identify differences in neural activity in relation to breathlessness,
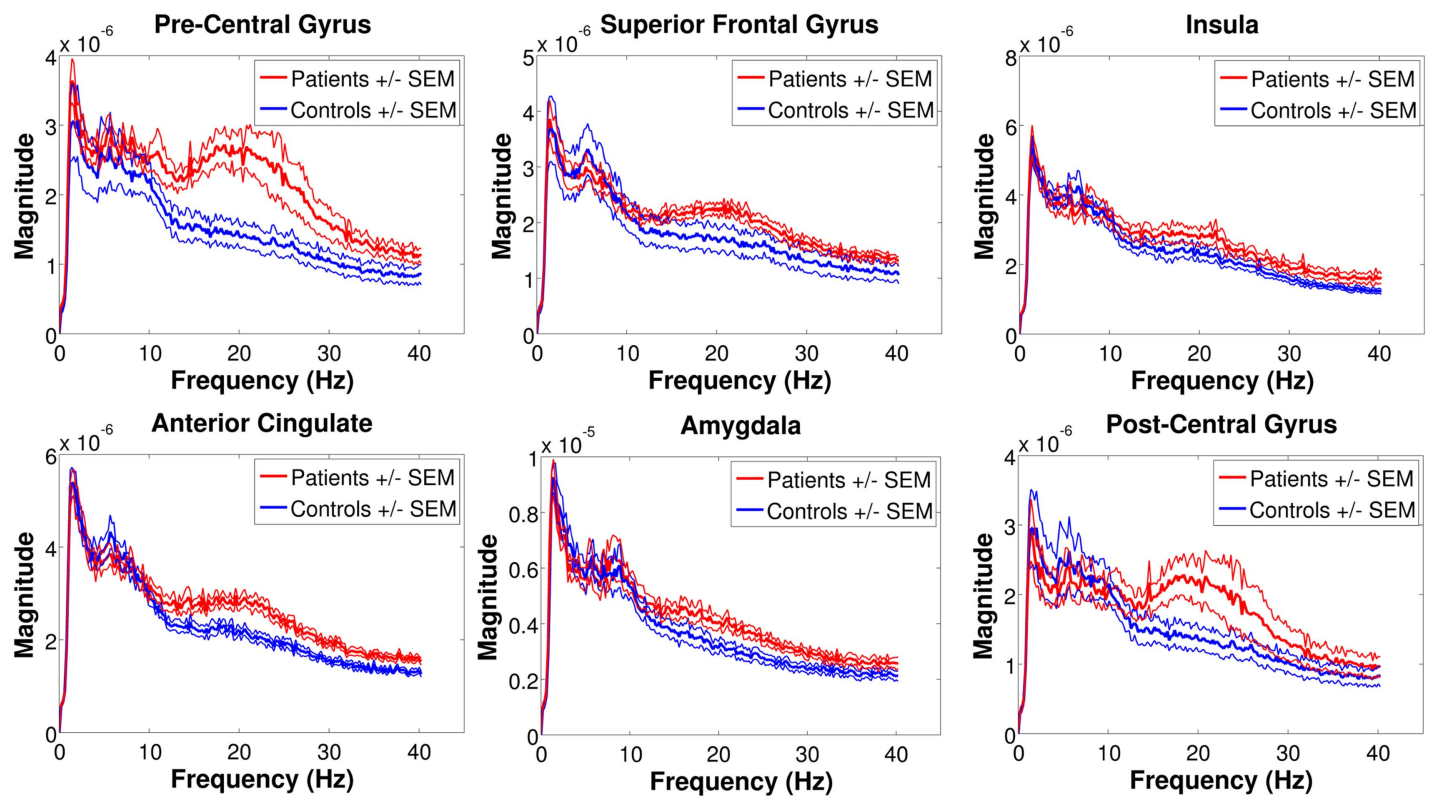

Figure 2 Resting data: participants and healthy (historical) controls. 
Table 1 Summary of brain activity by region according to breathless, or after recovery (bold black=breathless: grey non-bold=recovery).

\begin{tabular}{|c|c|c|c|c|c|c|c|c|c|c|c|c|c|}
\hline Pts & Region & $\boldsymbol{\alpha}$ & $\alpha$ (air) & $\alpha$ & $\alpha$ (air) & $\boldsymbol{\beta}$ & $\beta$ (air) & $\beta$ & $\beta$ (air) & $\gamma$ & $\gamma$ (air) & $\gamma$ & $\gamma$ (air) \\
\hline$\# 1$ & Frontal & + & + & - & - & + & + & & & + & + & + & + \\
\hline \#2 & & + & + & & - & + & - & - & - & - & - & + & + \\
\hline \#3 & & + & + & - & - & + & + & - & - & & + & - & - \\
\hline \#4 & & + & - & 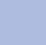 & - & + & + & + & - & - & + & + & \\
\hline \#5 & & + & + & - & - & + & + & - & - & + & + & + & - \\
\hline$\# 6$ & & + & + & - & - & + & + & - & - & + & & + & - \\
\hline$\# 7$ & & & + & - & - & + & - & - & & & + & & \\
\hline$\# 1$ & Temporal & - & - & + & + & + & + & & - & + & - & + & - \\
\hline \#2 & & - & + & - & & + & + & - & + & + & + & - & + \\
\hline \#3 & & + & - & + & + & + & + & - & - & & + & - & - \\
\hline$\# 4$ & & + & - & + & 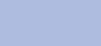 & + & + & + & + & - & + & + & \\
\hline \#5 & & + & - & + & + & + & + & + & + & + & + & + & + \\
\hline$\# 6$ & & + & + & - & - & + & + & + & + & + & & + & + \\
\hline$\# 7$ & & + & & - & - & + & + & - & & & + & & \\
\hline$\# 1$ & Posterior & - & - & . & + & - & - & & + & + & - & - & + \\
\hline \#2 & & - & + & + & & - & + & + & - & + & + & + & - \\
\hline \#3 & & - & - & + & + & - & - & + & + & & - & + & + \\
\hline$\# 4$ & & + & + & - & & - & + & - & - & + & + & - & \\
\hline \#5 & & - & - & + & + & - & - & - & - & - & - & + & + \\
\hline \#6 & & - & - & + & + & - & - & + & + & + & & + & + \\
\hline$\# 7$ & & & & + & nil & + & - & + & & & - & & \\
\hline
\end{tabular}

+ denotes that there are regions of activity where power is higher than in the alternate state.

-denotes that there are no regions of activity where power is higher than in the alternate state. Empty cells are shown where activity was neither increased nor decreased.

Pts, patients.

or the effect of airflow, however, these possible patterns raise hypotheses for further study. The following commentary on these data is therefore conjectural, and intended to stimulate discussion.

\section{Patterns of $\beta$ wave activity}

$\beta$ Wave activity was thought to represent the 'idling' motor state, ${ }^{26}$ and is the prominent activity seen at rest and awake. However, a more active role has been suggested whereby increased $\beta$ wave activity relates to active maintenance of the status quo (ie, suppression of change ${ }^{27}$ ) for motor and sensory activity. This is consistent with the somatosensory cortex being the dominant source of information flow in the $\beta$ band. ${ }^{28-30}$ For example, in Parkinson's disease, bradykinesia is associated with higher $\beta$ band activity, an abnormal persistence of the status quo and a loss of flexibility in the control of movement. ${ }^{31}$

Our data showed an increased $\beta$ band activity in the MNI coordinates associated with breathlessness perception in participants at rest compared with healthy controls. This may indicate constant 'vigilance', or an anticipatory state with regard to peripheral respiratory stimuli in chronic respiratory conditions. Furthermore, we observed a pattern whereby $\beta$ activity was higher in the frontotempoparietal regions when participants were breathless, but less on recovery. This could be consistent with the theory that increased $\beta$ activity is seen when there is a need to actively maintain the status quo; when breathless, the status quo is threatened by a strong endogeneous stimulus. With the addition of an exogeneous stimulus (eg, cool airflow), the expected pattern might be a reduction in $\beta$ band activity and increase in $\gamma ;{ }^{31}$ this was seen, but only in a few participants.

\section{Patterns of $\alpha$ wave activity}

$\alpha$ Waves functionally inhibit regions of the brain not required for attention, ${ }^{32}$ enabling sensory selection of attended stimuli. ${ }^{33}$ In a study of access to working memory, processing of distracters was distinguishable from the processing of items to be remembered; the higher the $\alpha$ activity, the better inhibition of distracters to gain access to working memory. ${ }^{34}$ Inhibition of task-irrelevant regions may allow rerouting of activity to relevant regions. ${ }^{35}$ This active processing in engaged areas is reflected by neuronal synchronisation in the $\gamma$ band which accompanies the $\alpha$ band decrease.

Our data show higher $\alpha$ wave activity in frontotemporal-parietal regions when breathless is compared with recovery. The clearest pattern is seen in the frontal regions (all participants). In the parietotemporal regions, this pattern appears to be modified by airflow. Given that participants described the airflow as pleasant, it may ameliorate unpleasantness by allowing sensory 


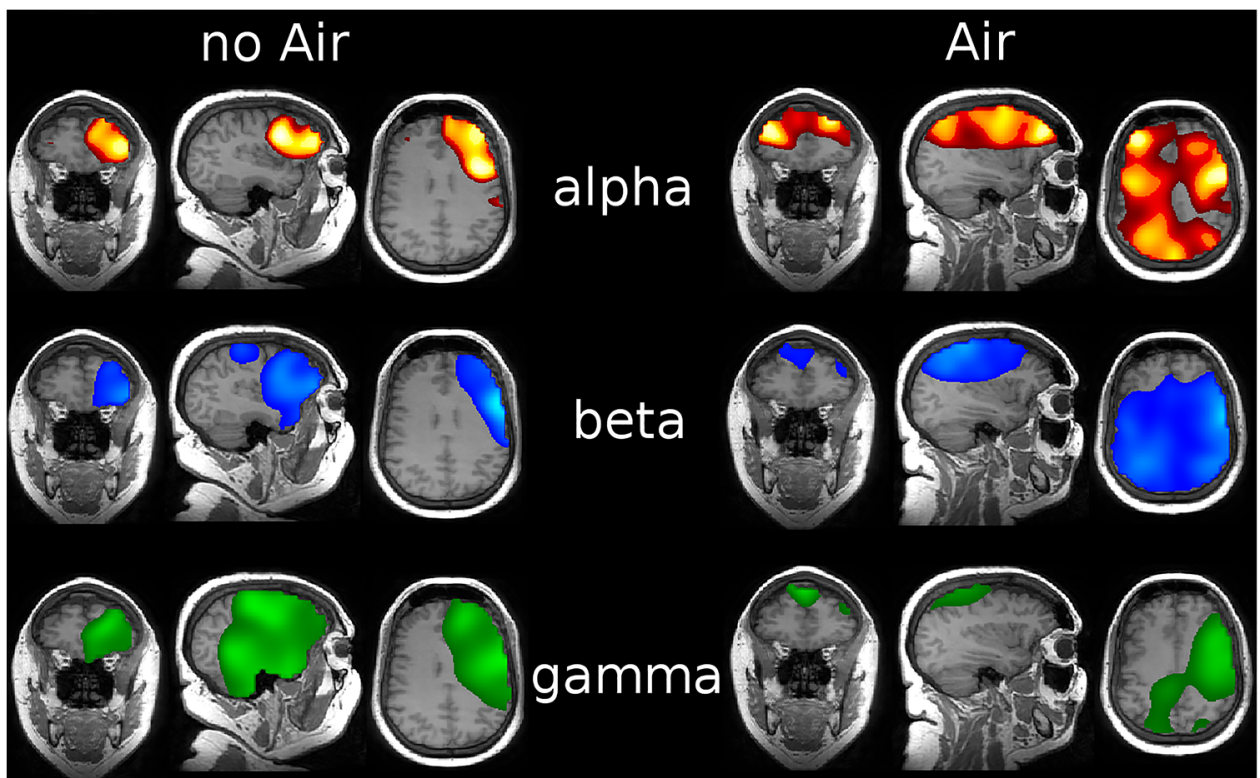

Figure 3 Beamforming activity postexercise >recovery, $\alpha, \beta, \gamma$ frequency bands. (The images are thresholded at $p<0.05$, and scaled using a hot colour scheme to their max-value). $\alpha$ Activity in the frontal lobes is clearly seen.

selection of a more pleasant stimulus. In a study of exercise-induced breathlessness in people with COPD, participants subjected to auditory distraction compared with no auditory distraction had reduced self-reported unpleasantness of breathlessness, although symptom intensity and distance walked were unchanged ${ }^{36}$ supporting sensory selection as a possible mechanism.

Our study showed an opposite pattern of brain activity in posterior regions; $\alpha$ activity was higher in participants after recovery than when breathless.

\section{Patterns of $\gamma$ wave activity}

$\gamma$ Waves are required for the modulation and processing of information arising simultaneously from different brain areas. They are thus crucial in conscious perception and foundational for all cortical computation. Our data suggest possible cross-frequency interaction between $\gamma$ and $\alpha$ when airflow is used when breathless ( $\alpha$ reduced in the frontoparietotemporal regions; $\gamma$ increased in the frontal regions). When breathless, airflow appears to increase $\gamma$ activity in the frontal regions, but reduces it in participants at recovery.

\section{Limitations}

Movement artifacts were not a problem at rest, however, all participants visibly moved during breathlessness. The uncertainty over the degree of movement is problematic, as not only do the source generators of peaks in the beamforming move over time in the scanner, but the confidence in the coregistration is also unknown during the scanning process itself. However, in view of this, to explore patterns of activity, we made broad estimates only of source localisation to 'within lobe'. Further
Figure 4 Beamforming activity recovery >postexercise, $\alpha, \beta$, $\gamma$ frequency bands. (The images are thresholded at $\mathrm{p}<0.05$, and scaled using a cool colour scheme to their max-value). $\alpha$ Wave activity is no longer seen in the frontal lobes.

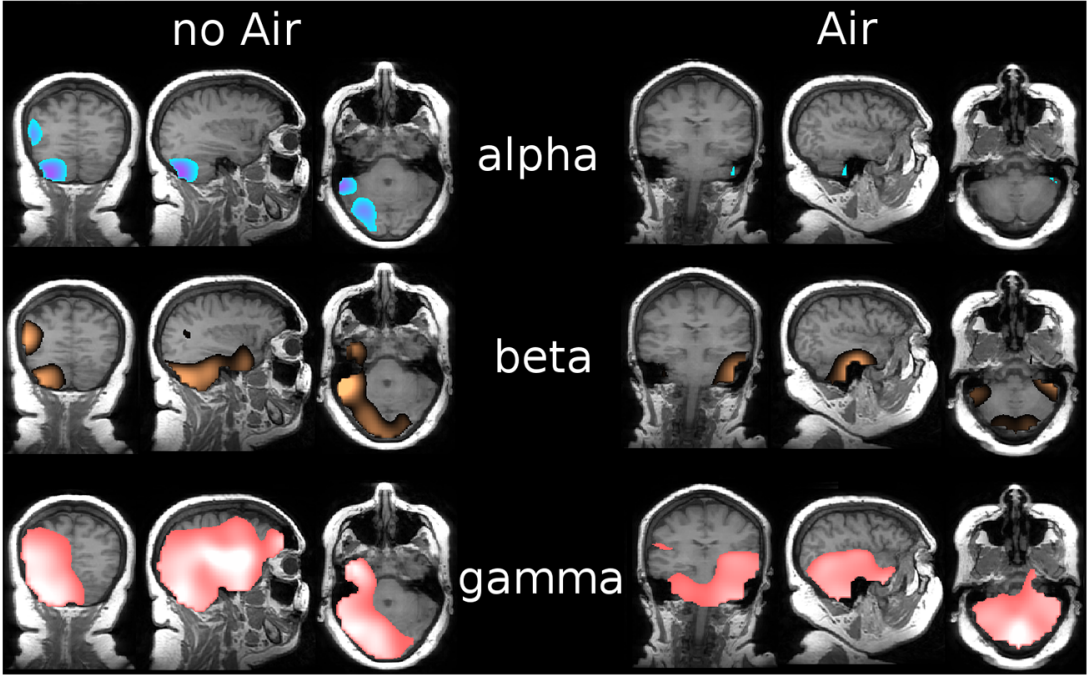


consideration of this difficulty and its possible solutions will be needed to draw more detailed location inferences. Various approaches such as real-time source estimation are described that can be taken into account in future work now we have demonstrated that patients with chronic lung disease can tolerate the procedures and that useful data are possible. ${ }^{37} 38$ Other methods of neural activity measurement such as EEG, for which head movement would not be an issue, should also be explored in future study in this area.

Our healthy controls were unmatched, and our findings could represent changes related to age or sex. However, one control appeared to have similar increased $\beta$ activity, and was subsequently removed from the analysis on discovery of a respiratory condition.

These data are from a small cohort with non-matched healthy controls. However, given the exploratory nature of the study, we feel that useful data has been acquired and our feasibility questions answered. The next step will be to confirm these results using a larger group with age and sex-matched controls. Thereafter, assuming confirmation, this technique could be used to explore the effects of interventions with clinical evidence to support their use in refractory breathlessness, such as airflow or opioids, on the central perception of this challenging symptom.

\section{CONCLUSIONS}

MEG scanning is a feasible, acceptable and potentially useful method to investigate breathlessness in people with chronic breathlessness. It is able to identify neural activity related to changes in breathlessness which can be induced by physical exertion.

Acknowledgements The authors thank Dr Ross Devlin, Lead Radiographer, York Neuroimaging Centre for help with MRI scanning.

Contributors MJ and GG conceived the question and design. MJ, GG and SH developed the protocol. MS, RM and MJ developed the procedure for the MEG scanning. MJJ, MIGS and REM collected the data, MIGS analysed the data, MJJ, MIGS, DCC and GG contributed to interpretation of the data; MJJ drafted the initial manuscript. All authors contributed to critical revision of the manuscript and agreed the final manuscript and agree to be accountable for all aspects of the work. MJJ had full access to all of the data in the study and takes responsibility for the integrity of the data and the accuracy of the data analysis.

\section{Funding Hull York Medical School Research Strategy Board.}

Competing interests MJ and DC are clinical advisors for Mayne Pharma.

Ethics approval National Research Ethical Service Committee Yorkshire and the Humber-Leeds East and the York Neuro-imaging Centre, University of York.

Provenance and peer review Not commissioned; externally peer reviewed.

Data sharing statement No additional data are available.

Open Access This is an Open Access article distributed in accordance with the Creative Commons Attribution Non Commercial (CC BY-NC 4.0) license, which permits others to distribute, remix, adapt, build upon this work noncommercially, and license their derivative works on different terms, provided the original work is properly cited and the use is non-commercial. See: http:// creativecommons.org/licenses/by-nc/4.0/

\section{REFERENCES}

1. Gysels M, Higginson IJ. Access to services for patients with chronic obstructive pulmonary disease: the invisibility of breathlessness. $J$ Pain Symptom Manage 2008;36:451-60.

2. Gysels M, Higginson I. Caring for a person in advanced illness and suffering from breathlessness at home: threats and resources. Palliat Support Care 2009:153-62.

3. Parshall MB, Schwartzstein RM, Adams L, et al. An official American Thoracic Society statement: update on the mechanisms, assessment, and management of dyspnea. Am J Respir Crit Care Med 2012;185:435-52.

4. Pattinson KT, Johnson MJ. Neuroimaging of central breathlessness mechanisms. Curr Opin Support Palliat Care 2014;8:225-33.

5. Von LA, Dahme B. Cortical substrates for the perception of dyspnea. Chest 2005;128:345-54.

6. Herigstad M, Hayen A, Wiech K, et al. Dyspnoea and the brain Respir Med 2011;105:809-17.

7. Von LA, Sommer T, Kegat S, et al. Down-regulation of insular cortex responses to dyspnea and pain in asthma. Am J Respir Crit Care Med 2009;180:232-8.

8. Von Leupoldt A, Brassen S, Baumann HJ, et al. Structural brain changes related to disease duration in patients with asthma. PLOS ONE 2011;6:e23739.

9. Hess A, Yu L, Klein I, et al. Neural mechanisms underlying breathing complexity. PLoS ONE 2013;8:e75740.

10. Proudfoot M, Woolrich MW, Nobre AC, et al. Magnetoencephalography. Pract Neurol 2014;14:336-43.

11. Ioannides AA. Magnetoencephalography as a research tool in neuroscience: state of the art. Neuroscientist 2006;12 524-44.

12. Galbraith $S$, Fagan $P$, Perkins $P$, et al. Does the use of a handheld fan improve chronic dyspnea? A randomized, controlled, crossover trial. J Pain Symptom Manage 2010;39:831-8.

13. Kerr D. A bedside fan for terminal dyspnoea. Am J Hosp Palliat Med 1989;6:22.

14. Schwartzstein RM, Lahive K, Pope A, et al. Cold facial stimulation reduces breathlessness induced in normal subjects. Am Rev Respir Dis 1987;136:58-61.

15. Abernethy AP, Mcdonald CF, Frith PA, et al. Effect of palliative oxygen versus room air in relief of breathlessness in patients with refractory dyspnoea: a double-blind, randomised controlled trial. Lancet 2010;376:784-93.

16. Maddocks M, Taylor V, Klezlova R, et al. When will I get my breath back? Recovery time of exercise-induced breathlessness in patients with thoracic cancer. Lung Cancer 2012;76:128-9.

17. Kozinska D, Carducci F, Nowinski K. Automatic alignment of EEG/ MEG and MRI data sets. Clin Neurophysiol 2001;112:1553-61.

18. Evans AC, Collins DL, Mills SR, et al. 3d Statis-Tical Neuroanatomical Models From 305 Mri Volumes. Proc IEEE-Nuclear Science Symposium And Medical Imaging Conference; 1993:1812-17.

19. Jenkinson $M$, Bannister $P$, Brady $M$, et al. Improved optimization for the robust and accurate linear registration and motion correction of brain images. Neuroimage 2002;17:825-41.

20. Van Veen BD, Van DW, Yuchtman M, et al. Localization of brain electrical activity via linearly constrained minimum variance spatial filtering. IEEE Trans Biomed Eng 1997;44:867-80.

21. Huang MX, Mosher JC, Leahy RM. A sensor-weighted overlapping-sphere head model and exhaustive head model comparison for MEG. Phys Med Biol 1999;44:423-40

22. Barnes GR, Hillebrand A. Statistical flattening of MEG beamformer images. Hum Brain Mapp 2003;18:1-12.

23. Pattinson KT, Mitsis GD, Harvey AK, et al. determination of the human brainstem respiratory control network and its cortical connections in vivo using functional and structural imaging. Neuroimage 2009;44:295-305.

24. Nichols TE, Holmes AP. nonparametric permutation tests for functional neuroimaging: a primer with examples. Hum Brain Mapp 2002;15:1-25.

25. Peiffer C, Costes N, Herve P, et al. Relief of dyspnea involves a characteristic brain activation and a specific quality of sensation. Am J Respir Crit Care Med 2008;177:440-9.

26. Pfurtscheller G, Stancak A Jr, Neuper C. Post-movement beta synchronization. a correlate of an idling motor area? Electroencephalogr Clin Neurophysiol 1996;98:281-93.

27. Kuhn AA, Williams D, Kupsch A, et al. Event-related beta desynchronization in human subthalamic nucleus correlates with motor performance. Brain 2004;127:735-46.

28. Zhang Y, Chen Y, Bressler SL, et al. Response preparation and inhibition: the role of the cortical sensorimotor beta rhythm. Neuroscience 2008;156:238-46. 
29. Tsujimoto T, Mima T, Shimazu H, et al. Directional organization of sensorimotor oscillatory activity related to the electromyogram in the monkey. Clin Neurophysiol 2009;120:1168-73.

30. Rubino D, Robbins KA, Hatsopoulos NG. Propagating waves mediate information transfer in the motor cortex. Nat Neurosci 2006;9:1549-57.

31. Engel AK, Fries P. Beta-band oscillations-signalling the status quo? Curr Opin Neurobiol 2010;20:156-65.

32. Foxe JJ, Snyder AC. The role of alpha-band brain oscillations as a sensory suppression mechanism during selective attention. Front Psychol 2011;2:154.

33. Handel BF, Haarmeier T, Jensen O. Alpha oscillations correlate with the successful inhibition of unattended stimuli. $J$ Cogn Neurosci 2011;23:2494-502.
34. Bonnefond $\mathrm{M}$, Jensen $\mathrm{O}$. Alpha oscillations serve to protect working memory maintenance against anticipated distracters. Curr Biol 2012;22:1969-74.

35. Jensen O, Mazaheri A. Shaping functional architecture by oscillatory alpha activity: gating by inhibition. Front Hum Neurosci 2010;4:186.

36. Von LA, Taube K, Schubert-Heukeshoven S, et al. Distractive auditory stimuli reduce the unpleasantness of dyspnea during exercise in patients with copd. Chest 2007;132:1506-12.

37. Little G, Boe S, Bardouille T. Head movement compensation in real-time magnetoencephalographic recordings. Methodsx 2014;1:275-82.

38. Vema Krishna MS, Maclellan M, Beyea S, et al. Faster and improved 3-D head digitization in MEG using Kinect. Front Neurosci 2014;8:326. 